\title{
AN ADAPTIVE REMOTE DISPLAY FRAMEWORK TO IMPROVE POWER EFFICIENCY
}

\author{
Dong Hyun, $\mathrm{Jo}^{1}$ and Dae Young, $\mathrm{Kim}^{2}$ \\ ${ }^{1}$ Department of Mobile Communication, \\ Samsung Electronics, Suwon, South Korea \\ donghyun. jodsamsung.com \\ ${ }^{2}$ Department of Computer Science, KAIST, Daejeon, South Korea \\ kimd@kaist.ac.kr
}

\begin{abstract}
As computing performance and network technology have evolved, mobile device users can enjoy high quality multimedia more easily. Remote Display - the technology which mirrors the screen of one device to another device - allows handheld mobile devices to share their screen contents with larger-sized display devices such as TVs. However, there is general concern about high power consumption caused by complex computation for encoding and continuous data transmission in the mobile devices.

In this paper, we present an adaptive remote display framework considering and utilizing the processing capability of display device. By supporting the Content Mirroring Mode, we can skip unnecessary steps and perform core activities to improve power efficiency and extend overall processing capability.
\end{abstract}

\section{KEYWORDS}

Remote display, Content Mirroring

\section{INTRODUCTION}

Recently, mobile devices such as smartphones and tablet PCs have become a part of everyday life. And advanced computing performance, high display resolution, and fast connectivity allow mobile device users to access and enjoy various multimedia services anytime, anywhere. Moreover, the cloud-based services provide appropriate content according to the type of connected device. Searching and downloading multimedia content that is compatible with the mobile device is no longer necessary.

However, in contrast with TV which has increasing screen size according to growing display resolution, the mobile devices which emphasize mobility and portability have restrictions in terms of screen size, so users are not able to maximize the experience of multimedia services. 
The remote display technology helps users to overcome the limit of screen size by providing a chance to mirror mobile device screen to another device with a large screen such as a TV. Using the remote display technology, users can also share the multimedia experience with family members or friends.

The visual quality and the end-to-end latency have been the technical challenges of the remote display. To mirror a high-resolution screen in real time, the screen has been captured and compressed using an encoding scheme to reduce the amount of data transfer. The efficient encoding scheme which provides better display quality and consumes lower network bandwidth has been researched [1].

Wi-Fi Miracast, a representative remote display solution, transmits screens encoded by H.264 video codec via peer-to-peer networking using a Wi-Fi direct (IEEE 802.11) connection. It provides good quality by supporting high resolutions of up to $1920 \times 1080$ pixels and low end-toend latency. However, whereas the high compression ratio of H.264 provides savings in network bandwidth, the high computational complexity of H.264 causes high power consumption and it still remains a problem on power-limited mobile devices.

Previous researches have mainly focused on an encoding scheme to improve the problem [2] and there has been a lack of interest in overall framework to solve the problem.

\section{RELATED WORKS}

Bo-yun Eom et al [1] have proposed a power-aware remote display framework which uses a hybrid encoding scheme in VNC protocol. It aims to improve power efficiency by switching encoding modes adaptively to the battery level of client devices.

Ji-su Ha et al [2] have proposed a scheme to implicitly analyse the dynamics of a video file and uses the screen dynamics score to compute an ideal frame rate in run-time with respect to the multimedia content context. The proposed work estimates the screen dynamics by calculating the I-type macroblocks in a target interval which can be configured and skips frames based on the normalized I-type macroblock count, the screen dynamics score. The video with low dynamics, like video lectures, have shown a lower screen dynamics score than a dance-genre music video. Using the screen dynamics score, which is related to the actual dynamics, the frame rate can be controlled to minimize the transmission and power consumption without visible quality loss.

There are several remote display technologies. Chih-Fan Hsu et al [3] have measured and compared the performance of those various technologies. The work provides the result of the performance evaluations in various aspects: frame rate, resolution, bitrate, packet loss and so forth. Most of them are proprietary solutions designed by manufacturers, which means it may not be compatible with some devices.

\section{BACKGROUND AND MOTIVATION}

A Remote display framework is comprised of three major parts: The source device which captures, encodes, and transmits the screen, the display device which decodes the received data and displays it, the data transmission protocol which defines the format of the data that is to be transmitted from a source device to a display device. 
The remote display is advantageous in that it can support any type of content if the source device can handle and display it. Conversely, the display device is responsible only for displaying a mirrored screen and the processing capability of display devices is not taken into account. However, various consumer electronics, such as TVs and refrigerators, have evolved into smart devices which have processing capability for various content and network connectivity [4]. We can utilize the display device as a content processing unit to extend the overall capability of the entire system.

The source device performs the intrinsic function which is content processing for local display. At the same time, it executes data processing and transmissions for remote display.

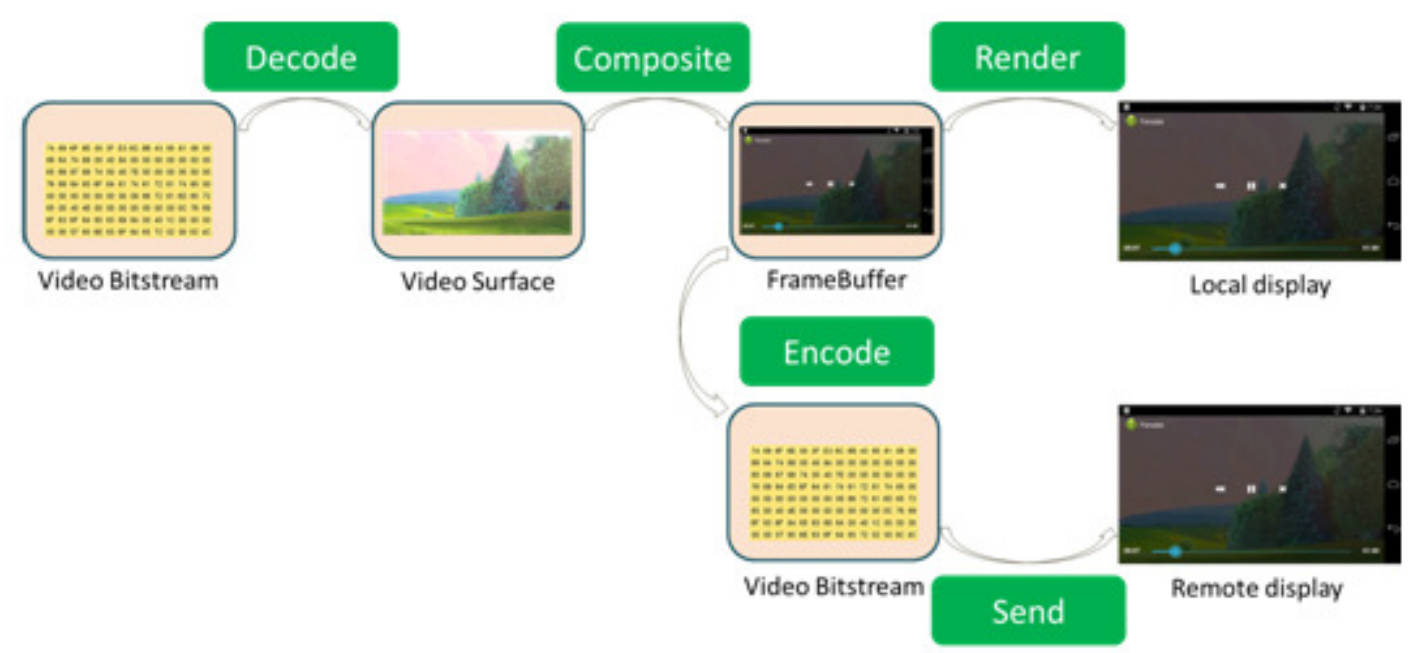

Figure 1. Process for the local display and the remote display

When playing a video, the following procedures are performed.

First, the media framework extracts the video bitstream from the media source and decodes it using the video decoder. Then, it passes the resulting video surface to the graphics engine. The video surface is resized to fit the render area. If necessary, the color format conversion is also carried out. The post-processed video surface is composited with a UI controller to make the final image for local display.

In addition to the above steps, the composited frame buffer is resized to fit the remote display and encoded to reduce network bandwidth. And then, it is sent to the remote display.

This intensive real-time processing results in lots of power consumption. Furthermore, in order to improve the visual quality of the remote display, more computation is required and more power consumption is generated. So, it is necessary to reduce and optimize the processes related to local and remote display.

In this paper, we present an approach considering an overall framework, including source device, data transmission protocol, and display device, from a broader perspective. As a result, we suggest a novel framework to improve power efficiency and extend overall processing capability for multimedia content. 


\section{ADAPTIVE REMOTE DISPLAY FRAMEWORK}

In Figure 1, the transmitted video bitstream is almost same as the original video bitstream except the composited UI controller, resolution and bitrate. To make the similar video bitstream for the remote display, power-consuming processes such as resizing and encoding are carried out. If it does not have to be exact same screen, it is possible to reduce the steps by sending the original video bitstream.

In certain scenarios, such as a media file playback and slide show, the source device works mainly as a controller, and the local display in the source device are not essential and generate unnecessary power consumption. We can consider it as an optional process and reduce power consumption of the source device by skipping these processes.

Adaptive remote display is based on the extended processing capability: The content can be processed in any device that is capable of handling it. In case of remote processing in which the content is processed in display device, the source device transmits the original content such as media file, streaming URI instead of encoded video stream. The display device processes the received content using its own framework and displays it. In addition, the source device is able to skip local processing and display, if necessary.

According to the transmitted data type, two modes are defined for data transmission protocol in adaptive remote display framework: the Screen Mirroring Mode (SMM) and the Content Mirroring Mode (CMM). The Screen Mirroring Mode is the same one used in typical remote display solution, which the source device process content and transfer the encoded screen to the display device.

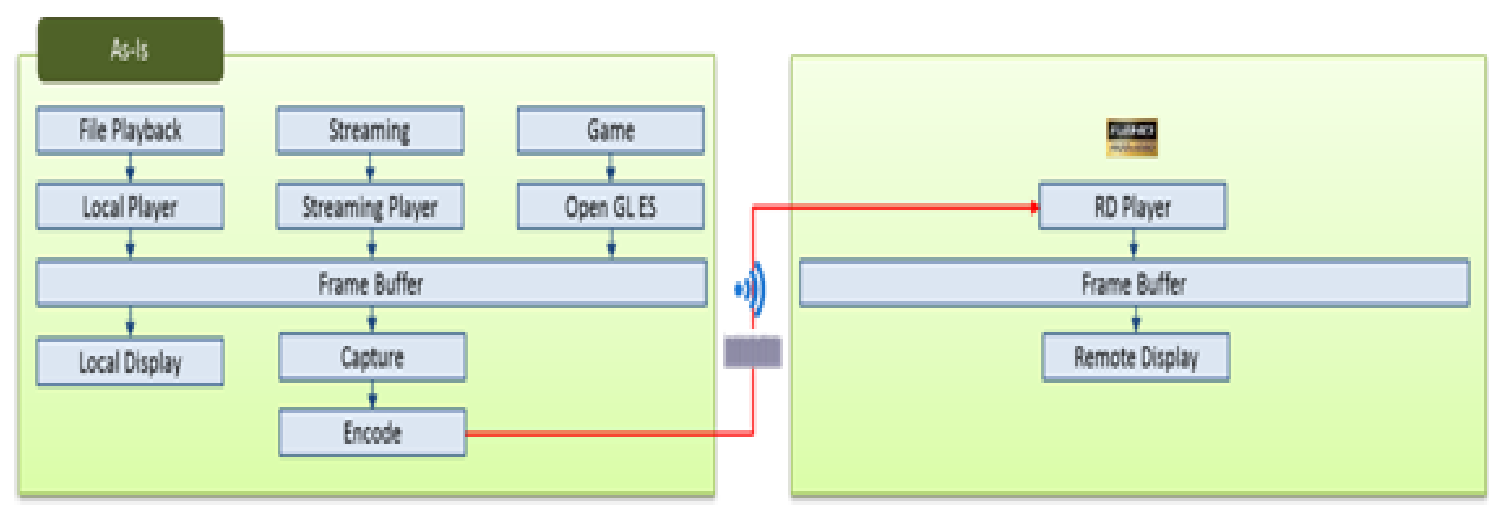

Figure 2. Block diagram for the Screen Mirroring Mode

In the Content Mirroring Mode, the content is transmitted instead of screen to the display device. In this mode, the frameworks are considered in an integrated way to support extended capability. 


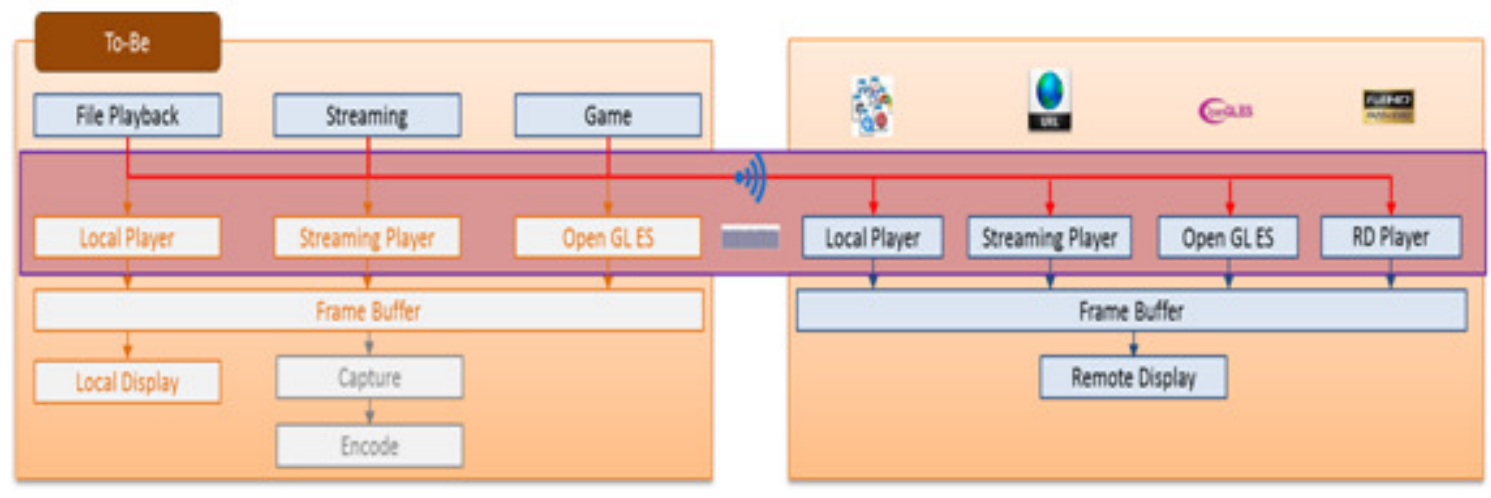

Figure 3. Block diagram for the Content Mirroring Mode

There are 3 main phases for the overall session of suggested solution.

\subsection{Processing Capability Negotiation}

Once a network connectivity completes successfully, the source and display device negotiate capability which both devices can process. The source device sends a request message to query the processing capability of the display device for contents such as media format/codec, streaming method, graphic library supported by the source device. The display device sends a response message listing contents that it is capable of processing, and then both devices finish the processing capability negotiation.

Table 1. Sample content for capability negotiation

\begin{tabular}{|l|l|}
\hline Capability & Example \\
\hline Text format & TXT \\
\hline Media format & 3GP, MP4, ASF, AVI, MKV \\
\hline Audio format & 3GA, M4A, WMA, MP3 \\
\hline Image format & GIF, PNG, BMP \\
\hline Streaming protocol & RTSP, HTTP, HTTP live streaming, \\
\hline Video codec & H.264, HEVC, MPEG4, VP8/VP9, \\
\hline Audio codec & LPCM, AAC, WMA, MP3, AMR, FLAC, DTS, AC3, RA \\
\hline Network access & Wi-Fi, LTE \\
\hline OpenGL version & 3.0 \\
\hline
\end{tabular}

If the source device is not capable of playing the AC3 audio codec and the display device has a AC3 decoder, the audio or video file can be played in the display device. In this way, the overall capability can be extended.

\subsection{Real-time Traffic Optimizer}

User can access contents in the source device while the display device is connected. First, based on the result of processing capability negotiation, adaptive remote display framework determines if currently accessed content can be processed by the display device. If the content is supported by the display device, one of the mirroring mode is selected based on the comparison result of the expected network bandwidth. If the content is not supported by the display device, this step is ignored and the Screen Mirroring Mode is kept. 
Table 2. An example of the network bandwidth comparison

\begin{tabular}{|l|l|l|}
\hline Content & Screen Mirroring Mode & Content Mirroring Mode \\
\hline HD 1.9 Mbps & $5 \mathrm{Mbps} * 2 \mathrm{~h} \mathrm{18m}=5.05 \mathrm{~GB}$ & $1.9 \mathrm{Mbps} * 2 \mathrm{~h} \mathrm{18m}=1.90 \mathrm{~GB}$ \\
\hline HD 5.4 Mbps & $5 \mathrm{Mbps} * 2 \mathrm{~h} 18 \mathrm{~m}=5.05 \mathrm{~GB}$ & $5.4 \mathrm{Mbps} * 2 \mathrm{~h} 18 \mathrm{~m}=5.42 \mathrm{~GB}$ \\
\hline
\end{tabular}

When playing the HD 1.9 Mbps video, the Content Mirroring Mode sends $1.90 \mathrm{~GB}$ and it saves $62 \%$ network bandwidth compared to the Screen Mirroring Mode. However, when playing the HD 5.4 Mbps video, the Screen Mirroring Mode shows advantage in network bandwidth. In this case, the overall power consumption including encoding and networking should be considered.

\subsection{Switching Mirroring Mode}

Once a mirroring mode is determined by the remote display framework, the source device informs the display device of new mirroring mode by sending the mode switching message. The message includes the type and detail information about the transmission data. For instance, when switching to video streaming, the message includes data type for video streaming and streaming URI. Once the display device receives the message, it unloads previous processing engine and loads appropriate processing engine.

\section{EVALUATIONS}

To evaluate the power consumption, Galaxy Note 5 (CPU: Quad-core $1.5 \mathrm{GHz}$ Cortex-A53 \& Quad-core 2.1 GHz Cortex-A57, GPU: Mali-T760MP8, Resolution: 1440 x 2560, Wi-Fi: 802.11 a / b / g / n, Android 5.1.1 Lollipop) and the Power Monitor (Monsoon Solutions Inc.) has been used. All the conditions including brightness, network connectivity have been controlled.

For the resolution and bitrate of the remote display, FHD (Full High Definition, 1920x1080) 10 Mbps and UHD (Ultra High Definition, 3840x2160) 10 Mbps have been used.

MX Player and YouTube have been used for local video playback and video streaming, respectively.

The various video clips have been selected for the evaluations.

Table 3. The test video clips

\begin{tabular}{|l|l|l|}
\hline Content & Properties of texture & Properties of movement \\
\hline $\begin{array}{l}\text { Nature } \\
\text { Food } \\
\text { Landscape }\end{array}$ & $\begin{array}{l}\text { Complexity: low } \\
\text { Texture change: mid }\end{array}$ & $\begin{array}{l}\text { Movement: low } \\
\text { Background moves slowly }\end{array}$ \\
\hline $\begin{array}{l}\text { Sport } \\
\text { Sea }\end{array}$ & $\begin{array}{l}\text { Complexity: mid } \\
\text { Texture change: high }\end{array}$ & Movement: high \\
\hline $\begin{array}{l}\text { Music Video } \\
\text { Movie }\end{array}$ & $\begin{array}{l}\text { Complexity: mid } \\
\text { Texture change: } \text { high }\end{array}$ & Movement: mid \\
\hline
\end{tabular}




\subsection{Screen Mirroring Mode vs. Content Mirroring Mode w/ local display}
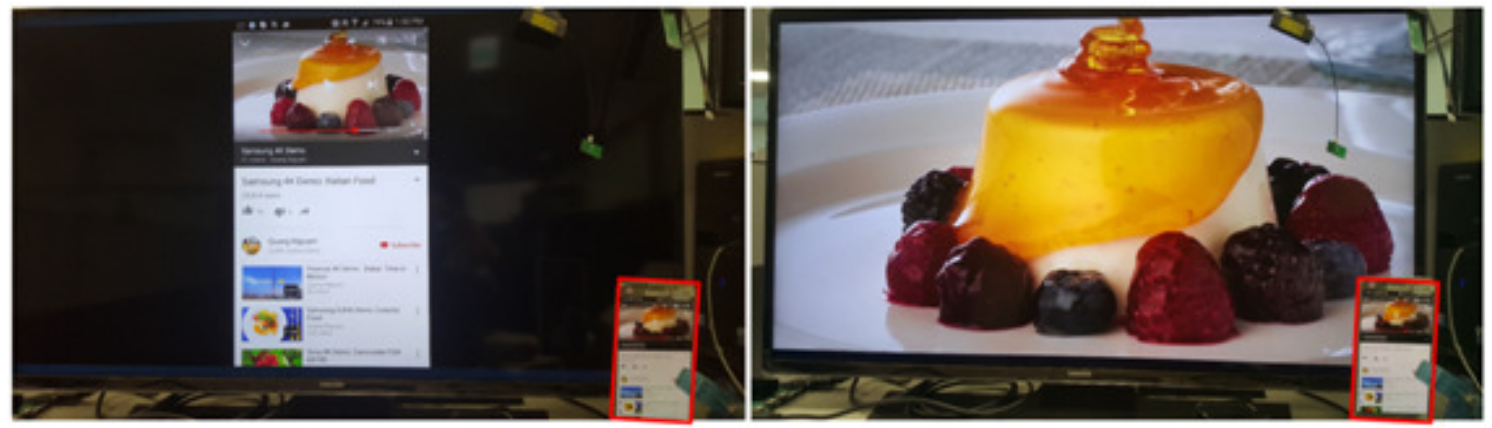

Figure 4. Screen Mirroring Mode (left), Content Mirroring Mode w/ local display (right)

As shown in Figure 4, the Screen Mirroring Mode mirrors the current screen to the remote display. The transmission data type is the encoded screen. So, it shows the same screen on the remote display.

The Content Mirroring Mode transmits raw data such as streaming URI, media file, and audio/video bitstream. The transmission data type is determined according to the capabilities of the display device.

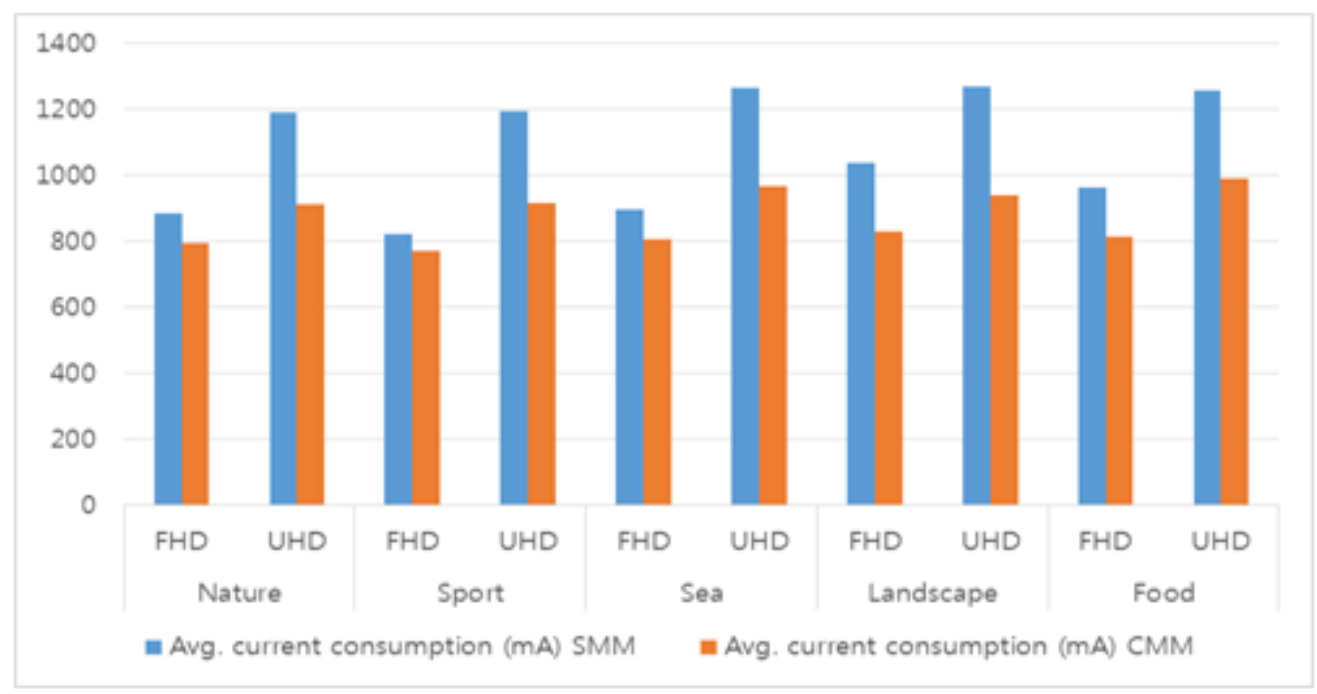

Figure 5. Comparison of the power (current) consumption:

Screen Mirroring Mode vs. Content Mirroring Mode (w/ local display, type: a/v bitstream)

Figure 5 shows the comparison result of the Screen Mirroring Mode and the Content Mirroring Mode which sends the a/v bitstream. In this case, the Content Mirroring Mode skips the resizing and encoding steps. The local display is also performed the same as the Screen Mirroring Mode.

The result shows a $12.4 \%$ (FHD) and $23.4 \%$ (UHD) improvement in power consumption. 


\subsection{Screen Mirroring Mode vs. Content Mirroring Mode w/o local display}
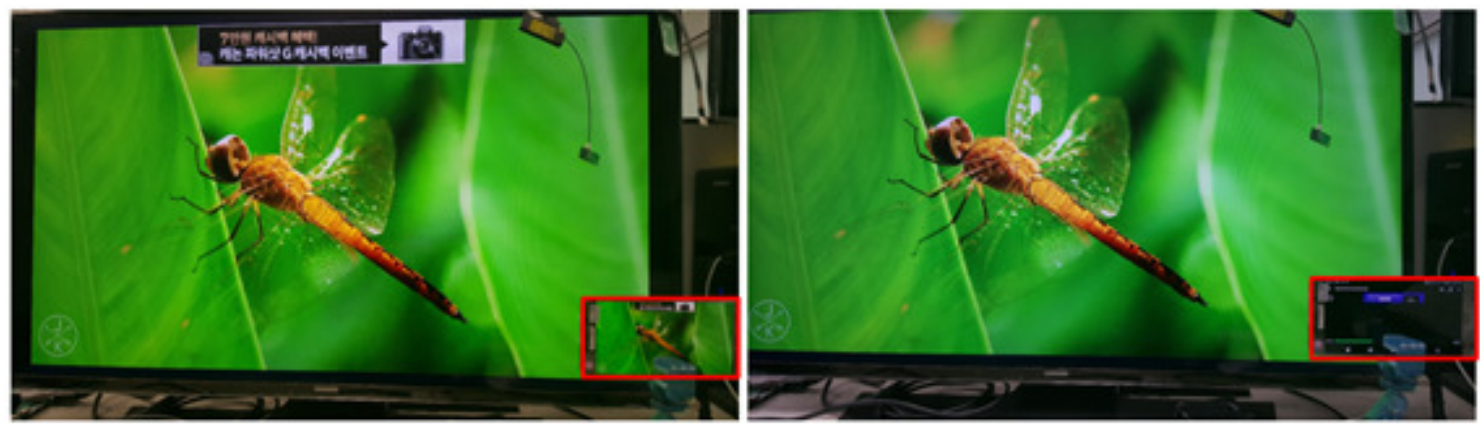

Figure 6. Screen Mirroring Mode (left), Content Mirroring Mode w/o local display (right)

When the user watches a video using the remote display technology, the local display may not be necessary because the video is played in the larger display. So the local display is a possible option we can skip. The Figure 6 shows the screen of the Screen Mirroring Mode which enables the local display and the screen of the Content Mirroring Mode which disables the local display. In this case, the power consumption caused by the local display can also be reduced in the Content Mirroring Mode.

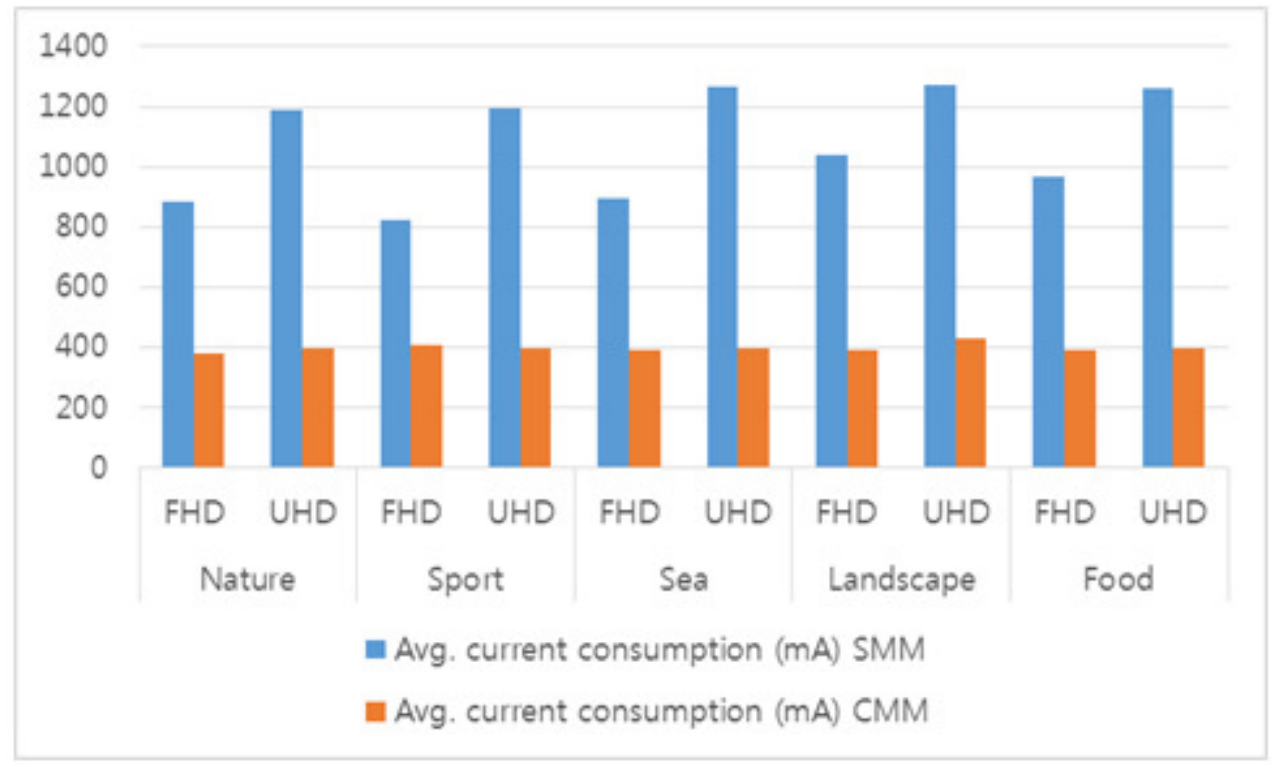

Figure 7. Comparison of the power (current) consumption:

Screen Mirroring Mode vs. Content Mirroring Mode (w/o local display, type: streaming URI)

When the display device is capable of processing streaming URI, which means it has a network connection such as wi-fi and it has an http streaming engine, the source device can transmit streaming URI. In this case, the source device skips the real-time processing for streaming video. As a result, the Content Mirroring Mode shows a $57.1 \%$ (FHD) and a $67.4 \%$ (UHD) improvement in power consumption. 


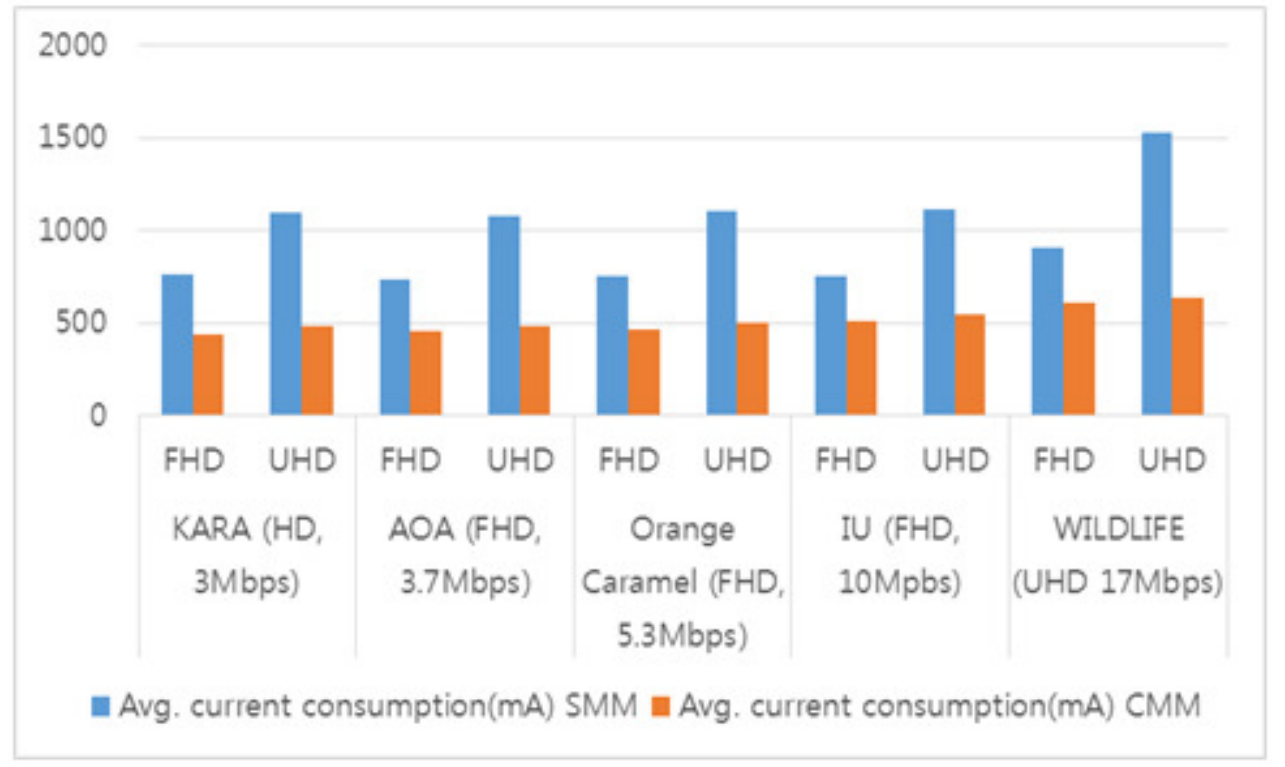

Figure 8. Comparison of the power (current) consumption:

Screen Mirroring Mode vs. Content Mirroring Mode (w/o local display, type: a/v bitstream)

The result shows a 36.5\% (FHD) and a 55.0\% (UHD) improvement in power consumption.

\subsection{Content Mirroring Mode w/ LCD on vs. Content Mirroring Mode w/ LCD off}

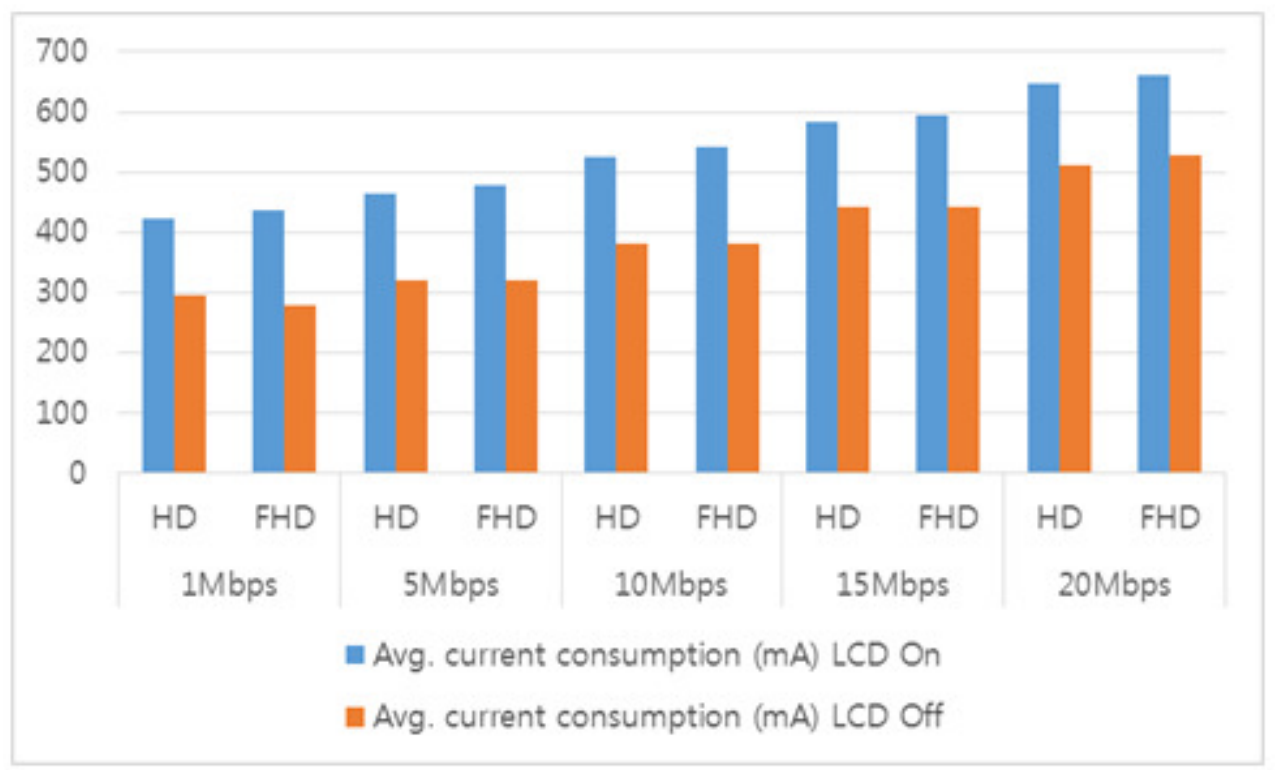

Figure 9. Comparison of the power (current) consumption:

Content Mirroring Mode (with LCD on) vs. Content Mirroring Mode (with LCD off) (w/o local display, type: a/v bitstream)

If the local display is not necessary, there is another advantage in the Content Mirroring Mode. It can turn the LCD of the source device off. When the user watches a movie which has long 
running time, this option saves the battery of the source device. When the LCD is off, the result shows an additional improvement of $27.8 \%$ in the power consumption is obtained.

\subsection{Content Mirroring Mode w/ bitrate change}

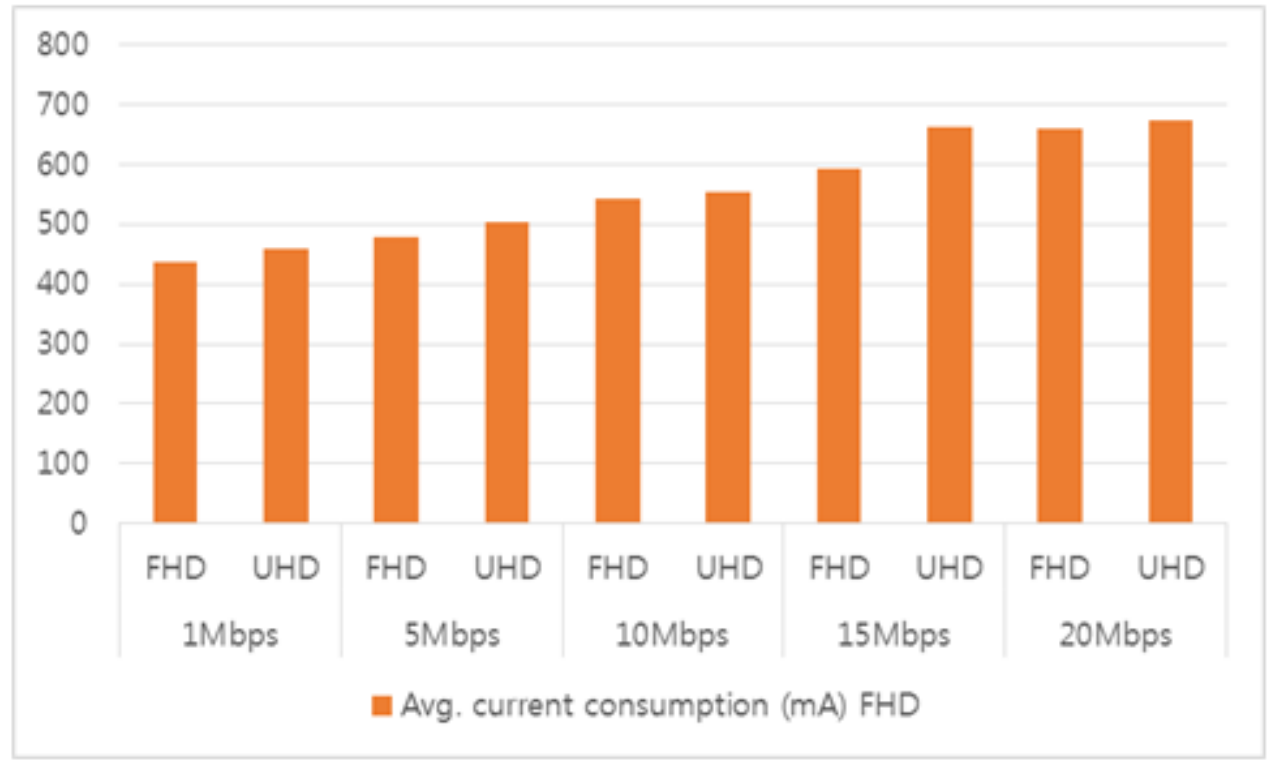

Figure 10. Comparison of the power (current) consumption: Content Mirroring Mode (w/o local display, type: a/v bitstream)

As the bitrate of a video file increases, the power consumption also increases for data transmission. The result shows that power consumption of $10.5 \%$ is added when the bitrate increases by $5 \mathrm{Mbps}$.

\section{CONCLUSIONS}

The remote display enables users to enjoy multimedia contents on a large screen. An adaptive remote display framework keeps the advantage and extends the overall capability. By reducing the content processing, the source device consumes lower power. Besides, in the Content Mirroring Mode, the amount of data transmitted can also be reduced. As a result, the power efficiency of mobile device is improved.

According to the evaluations, the Content Mirroring Mode which transmits a/v bitstream has shown a $12.4 \%$ (FHD $10 \mathrm{Mbps}$, w/ local display) to a 55\% (UHD $10 \mathrm{Mbps}$, w/o local display) improvement in power consumption compared to the Screen Mirroring Mode. In the case of transmitting the streaming URI, the Content Mirroring Mode shows a 57.1\% (FHD 10Mbps) and a $67.4 \%$ (UHD 10Mbps) improvement in power consumption. Furthermore, there is additional improvement of $27 \%$ in the power consumption if LCD is turned off, which is possible in the Content Mirroring Mode. 


\section{REFERENCES}

[1] Boyun Eom, Choonhwa Lee, "An adaptive remote display scheme to deliver mobile cloud services", IEEE Transaction Consumer Electronics, Vol. 60, Aug. 2014

[2] Jisu Ha, Puleum Bae, Keun-Woo Lim, JeongGil Ko, Young-Bae Ko, " Mobile contents on the big screen: adaptive frame filtering for mobile device screen sharing", Proceedings of the 12th ACM Conference on Embedded Network Sensor Systems, pp. 360-361, Nov. 2014

[3] Screencast in the Wild: Performance and Limitations, Chih-Fan Hsu and De-Yu Chen, ACM MM '14

[4] http://www.samsung.com/sec/SmartHome/sHome.html

[5] How is Energy Consumed in Smartphone Display Applications?, Xiang Chen, Yiran Chen, ACM HotMobile'13

[6] Profiling Power Consumption on Mobile Devices, Luca Ardito, Energy 2013

[7] Casual Mobile Screen Sharing, Proceedings of the Fifteenth Australasian User Interface Conference (AUIC2014)

[8] Cloud Mobile Media: Reflections and Outlook, IEEE Transactions on Multimedia, vol. 16, no. 4, June, 2014

[9] Mirror, Mirror, On The Wall: Collaborative Screen-Mirroring for Small Groups, TVX 2014, June, 2014

[10] Wi-Fi CERTIFIED Miracast ${ }^{\mathrm{TM}}$ : Extending the Wi-Fi experience to seamless video display, Wi-Fi Alliance, September, 2012 